\title{
Ganho de peso durante a gestação de adolescentes e baixo peso ao nascer
}

\author{
Caroline Ronchini FERREIRA ${ }^{1}$ \\ Daniela Cristina Megda MACIEL ${ }^{2}$ \\ Natália Melissa Martins de OLIVEIRA ${ }^{3}$ \\ Roberta Ribeiro SILVA ${ }^{4}$ \\ Rosangela da SILVA ${ }^{5 *}$
}

1 - Nutricionista. Mestranda em Biociências Aplicadas à Saúde pela Universidade Federal de Alfenas (UNIFAL-

MG). e-mail: carolronchini@yahoo.com.br

2 - Nutricionista pela Universidade Federal de Alfenas (UNIFAL-MG). e-mail: dannimaciel@bol.com.br

3- Nutricionista pela Universidade Federal de Alfenas (UNIFAL-MG). e-mail: natmelissa@ hotmail.com

4 - Nutricionista. Doutora pela Universidade Federal de Minas Gerais (UFMG). Professora Adjunta do curso de

nutrição da Universidade Federal de Alfenas (UNIFAL-MG). e-mail: betaribeiro@hotmail.com

5 - Nutricionista. Doutora pela Universidade Federal de São Paulo (UNIFESP/EPM). Professora Adjunta do

curso de nutrição da Universidade Federal de Alfenas (UNIFAL-MG). email: rsilvanutri@gmail.com

*Autor para correspondência: Profa Dra Rosangela da Silva

Universidade Federal de Alfenas (UNIFAL-MG). Rua Gabriel Monteiro da Silva, 700 - Alfenas-MG - 37130-

000 - Brasil. Fone: (35) 3299-1106 / 1391 (35) 88493615 (11) 9206-1963. E-mail: rsilvanutri@ gmail.com

Recebido em: 30/05/2013 - Aprovado em: 15/07/2013 - Disponibilizado em: 15/08/2013

\section{RESUMO}

Objetivo: Analisar a associação entre gestação na adolescência e baixo peso ao nascer no Município de Alterosa - MG. Métodos: A pesquisa foi realizada na Santa Casa de Misericórdia e tratou-se de estudo epidemiológico de caráter retrospectivo. Foram coletados dados dos prontuários médicos das gestantes adolescentes, parturientes dos anos de 2008 e 2009, tais como: idade materna, idade gestacional em que ocorreu o parto, peso prégestacional, ganho de peso durante a gestação, número de consultas pré-natais e peso do recém-nascido. Para se verificar a presença ou não de associação entre as variáveis independentes e o desfecho estudado, estimou-se a Odds Ratio (OR) bruta para cada associação de interesse, com os respectivos intervalos de confiança de $95 \%$ (IC 95\%). Utilizando-se o teste do qui-quadrado de Pearson (variáveis categóricas) e o teste t de Student (variáveis contínuas). Foi utilizado o programa estatístico SPSS 17.0. Em todos os testes, se considerou significante um p<0,05. Resultados: A média de idade foi de 17,5 anos; todas as gestantes tiveram seus partos realizados a termo; no início da gestação a média do IMC foi de $22,1 \mathrm{~kg} / \mathrm{m}^{2}$ e ao final de $26,6 \mathrm{~kg} / \mathrm{m}^{2} ; 38 \%$ das adolescentes apresentaram ganho de peso insuficiente e $84,9 \%$ delas realizaram 6 ou mais consultas. A média de peso ao nascer foi de $3.254 \mathrm{~g}$ e apenas 3,77\% dos RN apresentaram baixo peso. Somente o ganho de peso insuficiente durante a gestação apresentou correlação estatisticamente significante com o baixo peso ao nascer. Conclusão: Apesar da média de gestantes adolescentes no Município de Alterosa-MG ser maior que a média nacional, o número de recém-nascidos com baixo peso ficou bem abaixo da estimativa nacional.

Palavras-chave: gravidez na adolescência, baixo peso ao nascer, fatores de risco. 


\section{ABSTRACT}

Purpose: Examine the association between pregnancy in adolescence and low birth weight in the municipality of Alterosa - MG. Methods: The research was conducted at Santa Casa de Misericórdia, and this was a retrospective epidemiological study. Data were collected from medical records of adolescences pregnant of the years 2008 and 2009, such as maternal age, gestational age at delivery took place, pre-pregnancy weight, weight gain during pregnancy, number of prenatal visits and weight of the newborn. To verify the presence or absence of association between the independent variables and the outcome studied, we estimated the odds ratio (OR) for each gross association of interest, with confidence intervals of 95\% (95\% CI). Using the chi-square test (categorical variables) and Student's t test (continuous variables). We used SPSS 17.0. In all tests, were considered significant at $\mathrm{p}<0.05$. Results: The mean age was 17.5 years, all pregnant women had their deliveries at term, in early pregnancy the mean BMI was $22.1 \mathrm{~kg} / \mathrm{m} 2$ and $26.6 \mathrm{~kg} / \mathrm{m} 2$ in the end, $38 \%$ of adolescents had insufficient weight gain and $84.9 \%$ of them underwent six or more visits. The average birth weight was $3254 \mathrm{~g}$ and only $3.77 \%$ of the newborns were underweight. Only insufficient weight gain during pregnancy showed a statistically significant correlation with low birth weight. Conclusions: It was also observed that although the average pregnant teenagers in the municipality of Alterosa - MG be higher than the national average, the number of newborns with low birth weight were lower than the national estimate.

Keywords: adolescent pregnancy, low birth weight, risk factors.

\section{INTRODUÇÃO}

O ser humano apresenta um desenvolvimento peculiar denominado adolescência no qual ele passa por mudanças biopsicosexuais. Esse período se estende entre as idades de 10 a 19 anos, segundo a Organização Mundial da Saúde (OMS) (NASCIMENTO et al, 2011).

A influência sexual exercida pelos meios de comunicação se contrapõe a uma sociedade que ainda não aceita a prática sexual em idades precoces e conduz ao surgimento dos paradoxos da gravidez na adolescência (LIZZARELLI et al, 2009).

Vários estudos reconhecem que a gravidez neste período é um problema de saúde pública por restringir as possibilidades de exploração da identidade e preparação para o futuro profissional (DIAS \& TEIXEIRA, 2010).

Mães muito jovens são consideradas fisiologicamente imaturas para suportar as modificações ocorridas durante a gravidez (FRANCESCHINI et al, 2003; SILVA \& TONETE, 2006).

Além disso, acredita-se que a gestação nesta fase da vida pode propiciar danos no desenvolvimento do recémnascido, visto que há maior incidência de partos prematuros, baixo peso ao nascer, crescimento intrauterino restrito e outras complicações (SILVA \& TONETE, 2006). No tocante ao baixo peso ao nascer, vários fatores contribuem para a forte relação existente com a adolescência como a imaturidade do sistema reprodutivo 
e biológico, ganho de peso inadequado durante a gestação, além de fatores socioculturais como baixo nível socioeconômico e estilos de vida adotados pelas adolescentes (GAMA et al, 2001).

No município de Alterosa - MG foi constatado que no ano de 2008 as gestantes adolescentes participaram com 18,3\% dos partos enquanto que em 2009 esse número aumentou para $27,7 \%$, superando a média brasileira que varia de 14 a $22 \%$ (YAZLLE, 2006). Além disso, como o estudo analisa todas as gestantes adolescentes destes anos, e tendo em vista a alta prevalência deste evento no município, os resultados podem ser comparados e extrapolados para a análise dos riscos nesta população em nível nacional. Portanto, o estudo objetiva determinar os fatores de risco que corroboram para a ocorrência de baixo peso ao nascer entre as gestantes adolescentes.

\section{METODOLOGIA}

Tratou-se de um estudo descritivo, transversal, retrospectivo realizado no município de Alterosa, localizado no sul de Minas Gerais.

Foram incluídas no estudo todas as gestantes adolescentes, de idade igual ou inferior a 19 anos, parturientes dos anos de 2008 e 2009. Foram excluídos os prontuários pertencentes às gestantes que tiveram bebês natimorto ou gêmeos.

Os dados foram compilados dos prontuários médicos arquivados na Santa Casa de Misericórdia, sendo de interesse as seguintes variáveis: idade materna, estatura materna, peso pré-gestacional, ganho de peso durante a gestação, número de consultas pré-natais, idade gestacional em que ocorreu o parto e peso do recémnascido.

Para o diagnóstico do estado nutricional pré-gestacional, foi calculado o índice de massa corporal (IMC) sendo este classificado segundo os critérios do Instituto de Medicina dos Estados Unidos (IOM). O diagnóstico do estado nutricional gestacional foi realizado de acordo com o proposto pelo Ministério da Saúde. A avaliação do ganho de peso total durante a gestação também foi realizada segundo as recomendações do IOM (ATALAH et al, 1997; SISVAN, 2004; IOM, 1992).

Foi considerado satisfatório a realização de 6 ou mais consultas prénatais (COSTA, 1992). A idade gestacional em que ocorreu o parto foi calculada com base na data da última menstruação, a partir da qual os recém-nascidos foram classificados em pré-termo, termo ou póstermo (menor de 37 semanas, 37 a 42 semanas, maior de 42 semanas, respectivamente) (REZENDE \& MONTENEGRO, 2011). 
Para a classificação do peso ao nascer foi utilizado o critério da Organização Mundial de Saúde (OMS): baixo peso (menos de $2.500 \mathrm{~g}$ ), peso insuficiente $(2.500 \mathrm{~g}$ a $2.999 \mathrm{~g})$, peso adequado (3.000g a $3.999 \mathrm{~g})$ e excesso de peso (4000g ou mais) (LIMA \& SAMPAIO, 2004).

Para se verificar a presença ou não de associação entre as variáveis independentes e o desfecho estudado, estimou-se a Odds Ratio (OR) bruta para cada associação de interesse, com os respectivos intervalos de confiança de $95 \%$ (IC 95\%). Utilizando-se o teste do quiquadrado de Pearson (variáveis categóricas) e o teste $\mathrm{t}$ de Student (variáveis contínuas). Foi utilizado o programa estatístico SPSS 17.0. Em todos os testes, se considerou significante um $\mathrm{p}<0,05$.

O projeto de pesquisa foi aprovado pelo Comitê de Ética em Pesquisa da Universidade Federal de Alfenas/UNIFALMG, protocolo $\mathrm{n}^{\circ}$ 050/2010.

\section{RESULTADOS}

Na Santa Casa de Misericórdia de Alterosa - MG, nos anos de 2008 e 2009, ocorreram 290 partos sendo que destes 55 eram de gestantes adolescentes. Ao final, a amostra totalizou 53 gestantes, sendo que as demais não foram incluídas, pois sofreram aborto. Em alguns prontuários não havia todas as informações necessárias e consequentemente o número total de gestantes variou, para algumas variáveis.

A média de idade entre as gestantes foi de 17,5 $\pm 1,38$ anos (13-19 anos). Todas as gestantes tiveram seus partos realizados a termo.

Em relação ao estado nutricional das adolescentes, no início da gestação a média do IMC foi de $22,1 \pm 3,6 \mathrm{~kg} / \mathrm{m}^{2}$ e ao final de $26,6 \pm 3,6 \mathrm{~kg} / \mathrm{m}^{2}$, sendo observados 28,26\% (13/46) de baixo peso no início e $35,7 \%(15 / 42)$ ao final. Ao final da gestação 38\% (16/42) das adolescentes apresentaram ganho de peso insuficiente (Tabela 1).

Tabela 1 - Estado nutricional no inicio e final da gestação e o ganho ponderal ao final da gestação de adolescentes no município de Alterosa - MG, nos anos de 2008 e 2009.

\begin{tabular}{ccc}
\hline Variáveis & $\mathrm{n}$ & $\%$ \\
\hline Estado nutricional inicial $\S$ & & \\
Baixo peso & 13 & 28,26 \\
Eutrofia & 24 & 52,17 \\
Sobrepeso & 8 & 17,40 \\
Obesidade & 1 & 2,17 \\
Total & 46 & \\
Estado nutricional final $\S \S$ & & \\
Baixo Peso & 14 & 33,33 \\
Eutrofia & 18 & 42,85 \\
Sobrepeso & 9 & 21,42 \\
Obesidade & 1 & 2,38 \\
Total & 42 & \\
Ganho Ponderal & & \\
Insuficiente & 16 & 38,00 \\
Adequado & 19 & 45,33 \\
Excessivo & 7 & 16,67
\end{tabular}


Total

42

§ IMC pré-gestacional; §§ IMC ao final da gestação.

Fonte: Os autores.

Quanto ao número de consultas realizadas no pré-natal, verificou-se média de 7,2 $\pm 1,9$ consultas (2-10 consultas), sendo que $84,9 \%(45 / 53)$ das adolescentes realizaram 6 ou mais consultas (tabela 2).

Tabela 2. Consultas pré-natais e classificação do peso ao nascer dos recém-nascidos de adolescentes no município de Alterosa - MG, nos anos de 2008 e 2009.

\begin{tabular}{ccc}
\hline Variáveis & $\mathrm{n}$ & $\%$ \\
\hline Consultas $\S$ & 8 & 15,10 \\
6 ou mais & 45 & 84,90 \\
Total & 53 & \\
Peso ao nascer & & \\
Baixo peso & 2 & 3,77 \\
Peso insuficiente & 12 & 22,64 \\
Peso adequado & 35 & 66,05 \\
Excesso de peso & 4 & 7,54 \\
Total & 53 &
\end{tabular}

$\S$ número de consultas médicas realizadas no prénatal.

Fonte: Os autores.

A média de peso ao nascer foi de $3.254 \pm 4,44 \mathrm{~g}(2.000-4.200 \mathrm{~g})$ e $3,77 \%$ dos recém-nascidos nasceram com baixo peso (Tabela 2).

Quando se processou a análise univariada, as variáveis ganho de peso durante a gestação e IMC final, apresentaram correlação estatisticamente significante com o baixo peso ao nascer.
Porém, na análise multivariada, apenas o ganho se peso durante a gestação apresentou correlação significativa, sendo que a chance de baixo peso ao nascer aumenta 6,73 vezes com o ganho de peso insuficiente.

O peso ao nascer teve correlação estatisticamente significante somente com o ganho de peso da adolescente ao final da gestação $\mathrm{OR}=6,73 ; \mathrm{p}=0,029 ;(1,22-37,12)$.

\section{DISCUSSÃO}

Atualmente vem ocorrendo um déficit na taxa de fecundidade total e em contraposição um aumento na porcentagem de adolescentes grávidas (YAZLLE, 2006). $\mathrm{O}$ aumento de gestantes adolescentes no Município de Alterosa-MG, observado entre os anos de 2008 e 2009, confirma a atual transição epidemiológica.

Os recém-nascidos de mães adolescentes apresentam maior frequência de prematuridade, baixo-peso ao nascer e doenças perinatais. Associados a esses riscos existem outros fatores como baixa escolaridade, pré-natal inadequado ou não realizado, baixa condição socioeconômica e estado nutricional materno comprometido (NETO \& SEGRE, 2012).

Ao se analisar os dados antropométricos maternos do início da gestação, $47,82 \%$ das adolescentes apresentaram riscos inerentes ao estado nutricional pré-gravídico, englobando as 
gestantes com baixo peso e sobrepeso/obesidade, em outro estudo houve uma razão de $34,7 \%$ das gestantes com risco relativo ao estado nutricional. Contudo, no presente estudo, a média de peso ao nascer foi semelhante entre os grupos de risco e o grupo das gestantes eutróficas, achados discordantes do estudo no qual foi verificado que a adequação da relação peso pré-gestacional/estatura aumenta a diferença entre as médias de peso ao nascer (FRANCESCHINI et al, 2003; FURLAN, et al, 2003).

Em Curitiba, um estudo observou que $41 \%$ das adolescentes apresentaram ganho de peso insuficiente e houve associação com o baixo peso ao nascer e parto prematuro (SILVA, 2005). Esses resultados reforçam nossos achados.

Com relação ao acompanhamento pré-natal as gestantes estudadas tiveram acesso, no mínimo, a uma consulta prénatal, sendo que $84,9 \%$ realizaram um número satisfatório de consultas (COSTA, 1992). Assim, pode-se concluir que a disponibilidade e o acesso aos serviços primários de saúde no município estão satisfatórios, o que pode ter contribuído para a pequena parcela de baixo peso ao nascer e a ocorrência de partos a termo.

Ao se analisar o período de nascimento, todas as gestantes tiveram seus partos realizados a termo. Resultados diferentes foram observados em outros estudos, os quais observaram prematuridade em $17,8 \%$ e $22,9 \%$ dos recém-nascidos das mães adolescentes, respectivamente (ROCHA et al, 2006; SANTOS et al, 2009).

A média de peso ao nascer foi de $3254 \pm 4,44 \mathrm{~g}$, se aproximando muito do encontrado em estudo entre gestantes de baixa renda - 3223,8g (FRANCESCHINI et al, 2003). Ainda com relação à classificação do peso ao nascer, estudo realizado em Curitiba, mostrou que $10 \%$ dos recém-nascidos apresentaram baixo peso ao nascer e $36 \%$ peso insuficiente. Já neste estudo observou-se que apenas $3,77 \%$ dos recém-nascidos apresentaram baixo peso e $12 \%$ peso insuficiente. Esse achado foi menor que o observado no estudo anteriormente citado, representando um resultado satisfatório, porém o recémnascido com peso insuficiente é também vulnerável a repercussão de fatores ambientais e sociais e apresenta grande risco de morbidade e mortalidade durante $\mathrm{o}$ primeiro ano de vida (SILVA, 2005).

Em estudo associando os fatores ligados ao baixo peso ao nascer no Sudeste do Brasil, demostrou que as gestantes adolescentes contribuíram com 10,02\% dos partos e houve $15,1 \%$ de baixo peso ao nascer (SURITA et al, 2011).

Assim, embora a média de gestantes adolescentes no Município de Alterosa, no período do estudo, tenha sido 
maior que a média nacional (YAZLLE, 2006), observou-se baixo percentual de recém-nascidos com baixo peso.

O Fundo das Nações Unidas para a infância relatou as incidências globais do baixo peso ao nascer, sendo que na América Latina os percentuais variavam desde $5 \%$ no Chile até $21 \%$ no Haiti, com uma média de $10,35 \%$. Isso nos distancia muito dos países da Comunidade Europeia onde os dados apontam uma média de $6,4 \%$ e $7,7 \%$ na América do Norte (SILVA, 2012).

Com relação aos fatores previamente delineados como de risco, somente o ganho de peso insuficiente durante a gestação apresentou correlação significante com o baixo peso ao nascer.

\section{CONCLUSÃO}

A gestação na adolescência vem sendo descrita por muitos autores como preditora de desfechos negativos como o baixo peso ao nascer. A análise dos fatores de risco para a ocorrência do fenômeno permitiu verificar que no grupo das adolescentes, apenas o ganho de peso insuficiente durante a gestação apresentou correlação significativa com o baixo peso ao nascer, aumentando em 6,73 vezes a chance de ocorrer o evento.

Estudos de acompanhamento de gestantes adolescentes desde os primeiros meses de gestação, englobando não só aspectos biológicos como também sociais e psicológicos devem ser feitos na população brasileira, a fim de delinear, de forma mais efetiva, os fatores de risco que levam à maior taxa de baixo peso ao nascer entre esse grupo. O conhecimento dos fatores de risco mais críticos para o surgimento de complicações durante a gestação entre as adolescentes propiciará à equipe multiprofissional uma atuação mais eficaz durante o pré-natal minimizando os desfechos negativos que podem advir deste período.

\section{REFERÊNCIAS}

ATALAH, E.S.; CASTILLO, C.L.; SANTORO, R.C.; ALDEA, A.P.

Propuesta de um nuevo estándar de evaluación nutricional en embarazadas.

Revista Médica Chile. v.125, n.12, p.1429-1436, 1997.

COSTA, A.M. O PAIMS: uma política de assistência integral à saúde da mulher a ser resgatada. São Paulo, Comissão de Cidadania e Reprodução, 1992.

DIAS, A.C.G.; TEIXEIRA, M.A.P. Gravidez na adolescência: um olhar sobre um fenômeno complexo. Paideia. v.20, n.45, p.123-131, 2010. 
FRANCESCHINI, S.C.C.; PRIORE, S.E.;

PEQUENO, N.P.F.; SILVA, D.G.;

SIGULEM, D.M. Fatores de rico para

baixo peso ao nascer em gestantes de baixa

renda. Revista de Nutrição. v.16, n.2,

p.171-179. 2003.

FURLAN, J.P.; GUAZZELLI, C.A.F.;

PAPA, A.C.S.; QUINTINO, M.P.;

SOARES, R.V.P.; MATTAR, R. A

Influência do Estado Nutricional da

Adolescente Grávida sobre o Tipo de Parto

e o Peso do Recém-nascido. Revista

Brasileira de Ginecologia e Obstetrícia.

v.25, n.9, p.625-630. 2003.

GAMA, S.G.N.; SZWARCWALD, C.L.;

LEAL, M.C. A Gravidez na Adolescência como fator de risco para o baixo peso ao nascer no município do Rio de Janeiro, 1996 a 1998. Revista de Saúde Pública. v.35, p.74-80, 2001.

IOM (Institute of Medicine), National Academy of Sciences. Nutrition during pregnacy and lactation: an implementation guide. Washington (DC): The Academy, 1992.

LIMA, G.S.P.; SAMPAIO, H.A.C. Influência de fatores obstétricos, sócioeconômico e nutricional da gestante sobre o peso do RN, estudo realizado em uma maternidade em Teresina, Piauí. Revista

\section{Brasileira de Saúde Materno Infantil.}

v.4, n.3, p.253-261, 2004.

LIZZARELLI, P.M.; PATTA, M.C.;

RODRIGUES, R.; BOREZOWSKI, A.;

DUARTE, G. Resultados Perinatais e

Maternos de Gestantes Adolescentes.

Revista Brasileira de Medicina.v. 66, n.5,

p. 125-129, 2009.

NASCIMENTO, M.G.; XAVIER, P.F.;

SÁ, R.D.P. Adolescentes grávidas: a vivência no âmbito familiar e social.

Revista Adolescência e Saúde. v.8, n.4, p.41-47, 2011.

NETO, M.I.N.P.; SEGRE, C.A.M. Análise comparativa das gestações e da frequência de prematuridade e baixo peso ao nascer entre filhos de mães adolescentes e adultas.

Einstein. v.10, n.3, p.271-277, 2012.

REZENDE, J.F.; MONTENEGRO, C.A.B.

Obstetrícia Fundamental. 12ed. Rio de

Janeiro: Guanabara Koogan; 2011.

ROCHA, R.C.L.; SOUZA, E.;

GUAZZELLI, C.A.F.; FILHO, A.C.;

SOARES, E.P.S.; NOGUEIRA, E.S.

Prematuridade e baixo peso entre recém-

nascidos de adolescentes primíparas.

Revista Brasileira de Ginecologia e

Obstetrícia. v.28 n.9, p.530-535, 2006. 
SANTOS, G.H.N.; MARTINS, M.G.;

SOUSA, M.S.; BATALHA, S.J.C. Impacto

da idade materna sobre os resultados

perinatais e via de parto. Revista

Brasileira de Ginecologia e Obstetrícia.

v.31, n.7, p.326-334, 2009.

SILVA, A.F.F. Gestação na Adolescência:

Impacto do Estado Nutricional no Peso do

Recém-Nascido [tese de mestrado].

Curitiba: Universidade Federal do Paraná.

79 p. 2005.

SILVA, L.; TONETE, V.L.P. A gravidez na adolescência sob a perspectiva dos familiares: compartilhando projetos de vida e cuidado. Revista Latino

Americana de Enfermagem. v.14, n., p.199-206, 2006.

SILVA, T.R.S.R. Fatores de risco materno não biológicos para o baixo peso ao nascer na América Latina: revisão sistemática da literatura com meta-análise. Einstein. v.10, n.3, p.380-385, 2012.

SISVAN (Sistema de Vigilância Alimentar e Nutricional). Orientações básicas para coleta, o processamento e análise de dados e a informação em serviço de saúde.

Ministério da Saúde, Brasília-DF; 2004.

SURITA, F.G.C.; SUAREZ, M.B.B.;

SIANI, S.; SILVA, J.L.P. Fatores

associados ao baixo peso ao nascimento

entre adolescentes no Sudeste do Brasil.

Revista Brasileira de Ginecologia e

Obstetrícia. v.33, n.10, 286-291, 2011.

YAZLLE, M.E.H.D. Gravidez na

Adolescência. Revista Brasileira de

Ginecologia e Obstetrícia. v.28, n.8, p.443-445, 2006. 